\title{
"Proyecto arRISKu MUGAN": Materiales para la reducción de los riesgos asociados al consumo de drogas.
}

\author{
“arRISKu MUGAN Project”: Materials aimed for reducing risks associated with drug use.
}

\author{
Patricia Insúa Cerretani a , Marimar Lledó Saínz de Rozas b \\ a Dra. Psicología. Profesora titular de la UPV/EHU. Facultad de Psicología. Donostia-San Sebastián, \\ España. ${ }^{b}$ Dra. Sociología. Profesora adjunta UPV/EHU. Escuela Universitaria de Trabajo Social. Vitoria- \\ Gasteiz, España.
}

Correspondencia: Patricia Insúa Cerretani (patricia.insua@ehu.es)

Recibido: 13/08/2013; aceptado con modificaciones: 09/02/2014

\begin{abstract}
RESUMEN: Introducción: El Proyecto “arRISKu $M U G A N$ ” ha sido diseñado por las autoras en 2010, desde el Servicio de Psicología Aplicada de la UPV/ EHU, con el apoyo de la Dirección de Drogodependencias del Gobierno Vasco, la MILDT ${ }^{1}$ y los municipios de Irun, Hondarribia, Oiartzun y Hendaya y basándose en las conclusiones del estudio "Consumo de alcohol y otras drogas a ambos lados del Bidasoa. Diagnóstico y propuestas de actuación” (1). Material y método: A través de una metodología de investigación-acción, se inicia un proceso de dinamización social, que más allá de realizar valoraciones consultivas, pretende crear grupos de trabajo que puedan tomar decisiones que afecten al espacio transfronterizo, y al consumo de alcohol y otras drogas en sus zonas de ocio y tiempo libre. En el proyecto se implican profesionales de la salud mental, jóvenes y hosteleros/as, con el objetivo de reducir los riesgos asociados al consumo de alcohol y otras drogas.

Resultados: Se diseñaron materiales de prevención selectiva tanto en francés como castellano y euskera, para su distribución en bares y discotecas. Se trata de una colección de posavasos, que conforman un póster con un diseño tipo puzzle.

Conclusiones: El proyecto se planteó como un plan de intervención a medio-largo plazo, con continuidad en el tiempo, lo que exige el compromiso de las Instituciones y los agentes sociales de la zona.

PALABRAS CLAVE: Prevención selectiva, Drogas de abuso, Reducción de riesgos, Participación social, Jóvenes.
\end{abstract}

ABSTRACT: Introduction: The "arRISKu MUGAN" project was designed by the authors, who are attached to the Psychology Applied Service of the UPV/EHU in 2010, with funding from the Drug Dependence Office of the Basque Government, MILDT and the municipalities of Irun, Hondarribia, Oiartzun and Hendaye, and it is based on the findings of the study "Consumption of alcohol and other drugs on both sides of the Bidasoa River. Diagnosis and proposals for action" (1).

Material and method: Through the methodology of research-action, a process of social dynamization starts, which beyond realizing consultative valuations, it tries to create working groups that could take decisions that concern the cross-border space and the consumption of alcohol and other drugs, in their zones of leisure and free time. In the project there are involved professionals of mental health, young people and alcohol servers and owners, with the aim to reduce the risks associated with the consumption of alcohol and other drugs.

Results: The selective prevention materials created were designed for their distribution in bars and nightclubs, and consist of a series of 9 beer mats with information in French, Spanish and Basque, on reducing the risks associated with the consumption of alcohol or other drugs. A full collection of these beer mats makes up a poster with a puzzle-type design.

Conclusion: The project was conceived as a medium to long-term plan, with continuity over time, and needs the participation and involvement of the Institutions and the social partners in the area.

KEY WORDS: Selective Prevention, Street Drugs, Risk and Harm Reduction, Social Participation, Young adult.

1 Mission Interministérielle de lutte contre la drogue et les conduites addictives

\section{Agradecimientos}

Las autoras agradecen la colaboración de los dueños y trabajadores de Café Irún, Decibelia, Zona Límite, Street End, Bar Manolo, Cheers, The Corner y Truck que participaron en las reuniones de trabajo.

Asimismo, agradecen a todos los profesionales de prevención y asistencia en drogodependencias de ambos lados de la frontera (Osakidetza /SVS, Bizia - Medícins du Monde, AGIPAD, Askagintza, ANPAAG4, ARIT, ZUBIA, Collège Irandatz, ARSA y Hôspital Inter Communal de la Côte Basque) que revisaron los materiales y a los y las jóvenes que realizaron los grupos de discusión. El diseño de los materiales fue financiado por diferentes Instituciones: el Gobierno Vasco (a través de la Dirección de Drogodependencias), la Mildt (a través de la Asociación Bizia-Medecins du Monde) y los Ayuntamientos de Irún, Hondarribia y Oiartzun, a través de un contrato con el Servicio de Psicología Aplicada de la UPV/EHU. 


\section{Introducción}

El estudio "Consumo de alcohol y otras drogas a ambos lados del Bidasoa. Diagnóstico y propuestas de actuación” (1), reveló que los espacios en los que con mayor frecuencia se realizan consumos de alcohol y otras drogas y las conductas de riesgo asociadas, son los lugares de ocio que habitualmente frecuenta la juventud (bares, discotecas, etc.), junto a las "lonjas" o "locales" en el País Vasco (2), y su equivalente en el Estado francés, los "garajes". Estos son los lugares en los que, según las conclusiones de distintos estudios, se hacía necesaria una intervención preventiva y de reducción de los riesgos asociados al consumo (3-5).

Asimismo, el trabajo realizado mostró que la información que maneja la población (tanto la joven que está de fiesta en dichos espacios, como la adulta -sus padres, madres, educadores, etc.-), dista muchas veces de estar basada en la evidencia científica, y se apoya en criterios morales a los que la juventud, en general, es refractaria (6-8).

Esta realidad, no desconocida pero sí objetivada, es la justificación sobre la que se apoyó la realización de una serie de materiales preventivos y de reducción de los riesgos asociados al consumo de alcohol y otras drogas, basados en la evidencia científica, para jóvenes que alternan en dichos espacios de ocio.

Partiendo de que los riesgos en el uso de drogas dependen de múltiples factores relacionados tanto con el estado de ánimo y el estado físico, como con la cantidad consumida, las mezclas realizadas, el lugar de consumo, etc. (9), y entendiendo que en cualquier consumo se asumen ciertos riesgos para la salud, se acordó dirigir la propuesta de reducir las consecuencias negativas del consumo de sustancias, tanto hacia las personas que consumen drogas, como hacia aquellas con las que éstas se relacionan en los espacios de ocio $(8,10-12)$. La idea es compartir momentos, tanto de diversión como de reducción de riesgos, y transmitir el mensaje de que si alguien no se cuida por sí mismo/a, puede hacerlo por su amigo/a, su novio/a, su compañero/a de viaje, buscando aumentar el apoyo y la comunicación entre los pares, siguiendo diferentes modelos de intervención comunitaria en salud (8, 13-15).

Así, se pensó en el diseño y distribución de posavasos informativos en los locales en los que el consumo se realiza: bares de copas, discotecas, etc. En estos lugares se trabaja en conexión inmediata con jóvenes que consumen, por lo que también es importante la formación dirigida a propietarios y trabajadores/as de dichos locales $(15,6)$.

Implementar el proyecto en un espacio fronterizo, en la frontera o muga (en euskera) entre el Estado español y francés, exige también que se tenga en cuenta el contexto cultural en el que se producen los consumos y los riesgos asociados a él. La persona al pasar la "muga", está bajo otra Administración sanitaria, política, judicial, etc., y esta realidad, generalmente obviada por la cercanía y relaciones de 
vecindad, supone diferentes consecuencias que deben ser señaladas y conocidas (17-18).

Así, el proyecto "arRISKu MUGAN" surgió de la necesidad social de hacer frente a los riesgos asociados al consumo de drogas en los espacios de ocio transfronterizos, teniendo en cuenta las afinidades y diferencias políticas, culturales y sociales de la población objetivo de intervención. Esta característica es la que ha motivado e impulsado este proyecto y la que lo hace original y único.

El nombre arRISKu MUGAN, ideado por las autoras, hace referencia al juego semántico que se deriva de ambas palabras en euskera y su traducción al español ("el riesgo en la frontera"/"en la frontera del riesgo") y a la similitud ortográficofonética entre "riesgo", "risk", "risque" y "arrisku", que hace que se comprenda en cualquiera de los idiomas que coexisten en este espacio.

Los materiales se han diseñado para ser repartidos en castellano, euskera y francés, lo que da muestra de la complejidad cultural que el proyecto tuvo que tener en cuenta. A su vez, y como manera de evitar los mensajes directivos en los que la conducta es orientada desde arriba, este material pretende permitir la reflexión de la población destinataria, en la confianza de que reducir riesgos implica empoderamiento y responsabilidad en el autocuidado $(8,18)$.

El proyecto "arRISKu MUGAN" se plantea como un proyecto de investigación-acción, considerando el desarrollo de un Modelo Lógico, un Plan general de implementación y diferentes planes de acción (1: "Participación activa del personal de hostelería", (2: "Participación activa del grupo de jóvenes", y 3: "Participación activa del grupo de profesionales expertos en drogodependencias") (19-21), teniendo en cuenta que:

a) Debe ser un proyecto con base comunitaria, en cuyo diseño participen hosteleros/as con locales de ocio y diversión, profesionales que intervienen en drogodependencias en el espacio transfronterizo, y jóvenes que alternan en los municipios de Irún, Hondarribia y Oiartzun. Éstos últimos serán, además, la población destinataria final.

b) Estará basado en los principios de la Reducción de riesgos y daños asociados al consumo de drogas $(5,22)$.

c) Los mensajes utilizados deben ser directos, concretos y basados en la evidencia científica, haciendo especial hincapié en conseguir mensajes que puedan generar debate entre los distintos grupos de personas y plantear el proyecto como una herramienta privilegiada para una campaña de comunicación más amplia.

Despertar la curiosidad a través de un mensaje específico, es cada vez más difícil de lograr en la "sociedad de la atención" en la que vivimos. Por tanto, para conseguir captar la atención, los mensajes han de ser novedosos, originales, con capacidad de provocar y estimular el interés (23). 
Teniendo en cuenta la cantidad de información disponible actualmente sobre el tema drogas-drogodependencias, es importante seleccionar los contenidos a transmitir, así como el método, de forma que la población objetivo no se vea saturada.

\section{Objetivos}

El objetivo general ha sido crear materiales de prevención selectiva para la reducción de los riesgos asociados al consumo de alcohol y otras drogas en los contextos festivos y de ocio a ambos lados del Bidasoa, para jóvenes, adecuados a la evidencia científica, que puedan ser posteriormente distribuidos en hostelería.

Como objetivos específicos, el estudio se ha planteado:

a) Impulsar el espacio transfronterizo, teniendo en cuenta su especificidad, como lugar estratégico para la prevención selectiva del consumo de alcohol y otras drogas.

b) Promover la participación de todos los agentes sociales implicados en dicho espacio (hosteleros, jóvenes, y profesionales que intervienen en drogodependencias a ambos lados de la frontera), en el diseño y elaboración de los contenidos de los materiales, desde una metodología de trabajo comunitario.

c) Crear un material común para la población joven de ambos lados de la frontera entre los Estados francés y español, rompiendo mitos transmitidos generacionalmente, de cara a unificar los mensajes de prevención selectiva y reducción de riesgos, y diseñar estrategias de acción conjuntas.

\section{Material y Método}

Una vez revisada la bibliografía sobre las intervenciones en contextos de ocio $(10-12,18)$ y realizada la valoración de necesidades específicas (1), se eligió como elemento central de prevención selectiva, el posavasos, elemento habitual en hostelería y relacionado con la venta de alcohol, considerándolo el canal a través del cual se pretende establecer la relación entre el emisor y el destinatario. El hecho de que el personal de hostelería sea quien lo ofrecería junto con la bebida, convierte a este colectivo en parte activa en la transmisión de las ideas de prevención. Así, el acto se convierte en mensaje (24).

Se planteó que cada posavasos hiciera referencia a un aspecto relacionado con los riesgos que se asumen al consumir, unido a referencias de la música y la 
literatura española, euskalduna y francesa, que permitan inferir los significados que se pretende transmitir con la información científica. A través de esta forma de discurso analógico, se aumenta la potencia comunicativa, ya que la referencia literaria permite hacer llegar a la población lo que se dice y lo que no se dice, el texto y el contexto (25-26), a la vez que saca el discurso del consumo de drogas de lo marginal, legitimando que las personas compartan no sólo las drogas y la fiesta, sino también la cultura.

En primer lugar, y como base para el trabajo definitivo, se realizó un primer borrador de los materiales (9 posavasos), previa revisión de la literatura sobre ocio nocturno y los últimos informes de la Comisión Clínica de la Delegación del Gobierno para el Plan Nacional sobre Drogas (27-31), con el fin de asegurar que los contenidos teóricos incluidos en cada uno de los posavasos, tuvieran la evidencia científica que los sustente. En la medida en que cada uno de los posavasos giraba en torno a un tema concreto (comorbilidad (32-33), agresividad ligada al consumo de drogas (30-31,34), riesgos asociados a la conducta sexual (35-36), efectos de las mezclas de sustancias (27-31), legalidad y uso de drogas a ambos lados de la frontera (37-38), etc.) se buscó el apoyo bibliográfico que sustentaba las afirmaciones que en ellos se vertían.

Posteriormente, y teniendo en cuenta cada uno de los Planes de acción diseñados como componentes del Programa global, se encadenaron una serie de acciones para la coordinación del trabajo comunitario $(19-20,39)$ tanto con representantes de hostelería, como con profesionales que intervienen en drogodependencias a ambos lados de la frontera, como con jóvenes que comparten espacios de ocio y tiempo libre en la zona transfronteriza.

Para el Plan de acción 1: "Participación activa del personal de hostelería" y a través del contacto con representantes de las Asociaciones de hosteleros/as de la zona, se ha llegado a dueños, encargados y trabajadores de diferentes establecimientos de hostelería y de ocio nocturno, y se ha podido promover la concienciación frente al problema del consumo de alcohol y otras drogas, a pesar de la cierta disonancia cognitiva que se creaba al pensar en disminuir los riesgos del consumo entre quienes viven de la venta de alcohol. El trabajo ha partido de la necesidad de establecer y concretar las posibilidades realistas de hacer prevención y reducción de riesgos en estos locales, clarificando y consensuando el tipo de implicación al que se compromete cada uno/a a la hora de poner en marcha acciones concretas. Asimismo, y como forma de reforzar su participación y dar garantía al proyecto, se ofreció formación específica y asesoramiento continuado sobre el tema al personal de hostelería implicado. Estas personas, dueñas de establecimientos de ocio, actuaron como grupo de "expertos/as", participando activamente en el proceso de diseño, creación y mejora de los materiales del Programa.

Para el Plan de acción 2: "Participación activa del grupo de jóvenes" se organizaron diferentes grupos de discusión entre jóvenes que alternan en el espacio 
transfronterizo, con el fin de evaluar los materiales elaborados, coordinados por alumnas del Practicum de la Facultad de Psicología de la UPV/EHU, supervisadas por las autoras. Los/as participantes han contribuido con sus opiniones a la mejora del formato y del lenguaje empleado.

Para el Plan de acción 3: "Participación activa del grupo de profesionales expertos en drogodependencias" se invitó a participar a profesionales de la prevención y la atención a las drogodependencias de ambos lados de la frontera, tanto de Servicios públicos y concertados como de Asociaciones que, en Francia, llevan la mayor parte de la atención y la prevención en adicciones. Se organizaron diferentes grupos de trabajo, que permitieron validar el contenido científico incluido en los mensajes, introduciendo también propuestas de mejora.

\section{Resultados}

a) Del Plan de acción 1: "Participación activa del personal de hostelería"

Si bien en un principio las personas que accedieron a participar en el proyecto, expresaron sus reservas con respecto al impacto de las campañas en la conducta de sus clientes, señalando que les parecía más adecuado trabajar en los Centros educativos, desde el trabajo grupal comprendieron la necesidad de su participación dentro de un proyecto más global.

Se realizaron 3 grupos de trabajo de 3 horas de duración cada uno, con 10 dueños de bares de noche y discotecas de la zona, que han dado como resultado del proceso participativo, las siguientes sugerencias:

a) Que el proyecto global se presente en algún macroevento musical, aunque no fuera en la zona transfronteriza, antes que en los bares y discotecas, de forma que se rompa la identificación entre bares/discotecas y consumo de drogas.

b) Que se negocie algún tipo de beneficio no económico (por ejemplo, la presencia de taxis por las noches para evitar que se conduzcan coches particulares, la disposición de autobuses interurbanos por la noche, la ampliación (media hora más) de la hora de cierre por la noche, o las actuaciones discretas de los efectivos policiales en aparcamientos, locales y zonas de bares, disuasorias del consumo/ venta/etc. de sustancias, evitando los dispositivos "de película").

c) Que los contenidos de los posavasos no incluyan información lesiva para sus intereses económicos.

El personal de hostelería recibió información básica acerca de los contenidos científicos, de cara a poder explicarlos a la clientela, en caso de ser consultados sobre los mismos. En este sentido, sus propuestas finales plantean: 
1. Que se incluya en los posavasos una dirección de Internet desde la cual quien lo desee, pueda completar la información que presentan.

2. Que se incentive a quienes consulten la web, con el sorteo de un regalo apetecible (un teléfono móvil, una tableta, etc.).

3. Que se incorpore en algún posavasos, información sobre los riesgos del botellón, y sobre las ventajas de consumir dentro de los bares (seguridad, higiene, etc.).

4. Que otros comerciantes que venden alcohol y tabaco (supermercados, tiendas, estancos, etc.), participen también en el proyecto.

Además, se plantea que los posavasos puedan estar plastificados, de manera que sean más funcionales en los locales, evitando que se estropeen si se mojan y aumentando su atractivo para ser coleccionados. Se propone incluir tarjetas de plástico de fidelización (tipo tarjeta de crédito), con el nombre del Programa de un lado y las frases literarias del otro, y en el color del posavasos en cuestión (ver tabla 1).

Tabla 1.

Criterios de mejora establecidos en el Plan de acción 1.

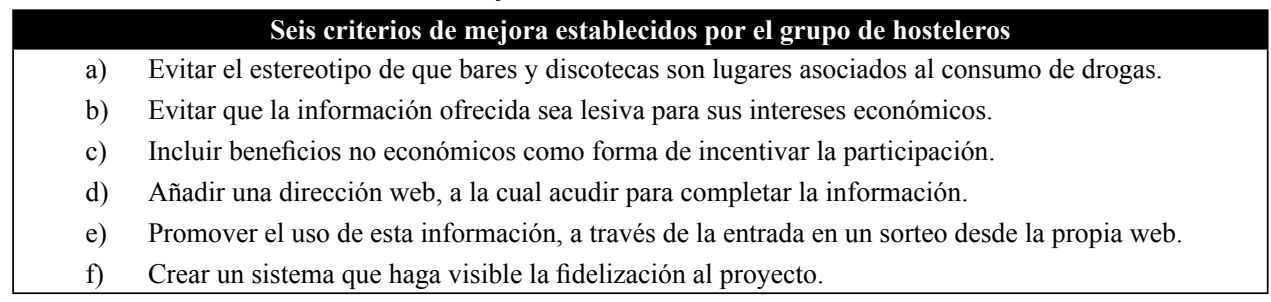

\section{b) Del Plan de acción 2: "Participación activa del grupo de jóvenes"}

Se realizaron 3 grupos de discusión de 3 horas de duración cada uno de ellos, con 10 jóvenes cada uno y las aportaciones propuestas han tenido que ver, fundamentalmente, con la necesidad de simplificar el lenguaje utilizado manteniendo la información científica.

La opinión general sobre el proyecto ha sido muy positiva. Los/as jóvenes, además, han propuesto la realización de un póster con los contenidos teóricos del reverso de los posavasos (no sólo de las caras que contienen las citas literarias), ya que ello permitiría leer todos los contenidos, y promoverlos como material coleccionable.

Por otra parte, se ha sugerido que los posavasos se dejen al alcance de todas las personas, sin que tenga que ser el personal del bar el que lo facilite. Sus conclusiones más relevantes se señalan en la tabla 2 (ver tabla 2 ). 
Tabla 2:

Criterios de mejora establecidos en el Plan de acción 2.

Siete criterios de mejora establecidos por el grupo de jóvenes

a) Simplificar el lenguaje.

b) Reducir la cantidad de texto.

c) Introducir datos estadísticos de impacto realista.

d) Incluir información más específica que general, en cuanto a las drogas y su consumo.

e) Eliminar expresiones ambiguas.

f) Evitar mensajes que puedan incitar al consumo, de forma no intencionada.

g) Incluir información sobre los recursos existentes en caso de tener problemas con el consumo de drogas, así como en caso de buscar más información.

c) Del Plan de acción 3: "Participación activa del grupo de profesionales expertos en drogodependencias"

Se realizaron 4 grupos de trabajo de 4 horas de duración cada uno, con entre 15 y 21 profesionales, uno de los cuáles fue monográfico sobre los materiales diseñados. Entre las principales aportaciones recogidas en las reuniones realizadas con el grupo de profesionales expertos, se pueden señalar la pertinencia de ofrecer información complementaria a la ofrecida en lo posavasos, la utilización de un lenguaje cercano pero que no banalice la información científica, y la necesidad de destacar el teléfono de Emergencias, para que se recuerde dónde llamar en caso de necesidad (ver tabla 3 ).

Tabla 3.

Criterios de mejora establecidos en el Plan de acción 3.

\section{Ocho criterios de mejora establecidos por el grupo de profesionales}

a) Incluir la dirección de una página web asociada, en la que se pudiera encontrar información complementaria.

b) Utilizar un lenguaje compartido con los/as jóvenes pero que no banalice la información científica.

c) Editar los materiales en cada uno de los tres idiomas del espacio transfronterizo.

d) Utilizar citas literarias propias de las diferentes culturas que conviven en el espacio transfronterizo y no traducirlas.

e) Incluir el teléfono de Emergencias.

f) Incluir en la página web asociada al proyecto, juegos, sorteos, concursos.

g) Distribuir los posavasos de forma progresiva, asociando campañas municipales que refuercen los mensajes a nivel comunitario.

h) Implicar a Centros comerciales y supermercados en la publicidad del proyecto. 
ORIGINALES Y REVISIONES

\section{Discusión}

El proyecto “arRISKu MUGAN”, está compuesto finalmente por 9 posavasos en español, euskera y francés con diferentes mensajes relacionados con los riesgos asociados con: a) conducir bajo los efectos del alcohol u otras drogas; b) la vulnerabilidad genética para la adicción; c) la mezcla de sustancias; d) las prácticas sexuales bajo el efecto del alcohol u otras drogas; e) la agresividad secundaria al consumo de drogas; f) la responsabilidad de los amigos/as en el abuso de sustancias; g) consumir alcohol u otras drogas como respuesta ante el estrés; h) la adicción en general; i) las consecuencias jurídicas derivadas del consumo de alcohol u otras drogas y sus diferencias en los dos Estados transfronterizos (ver figuras 1 y 2).

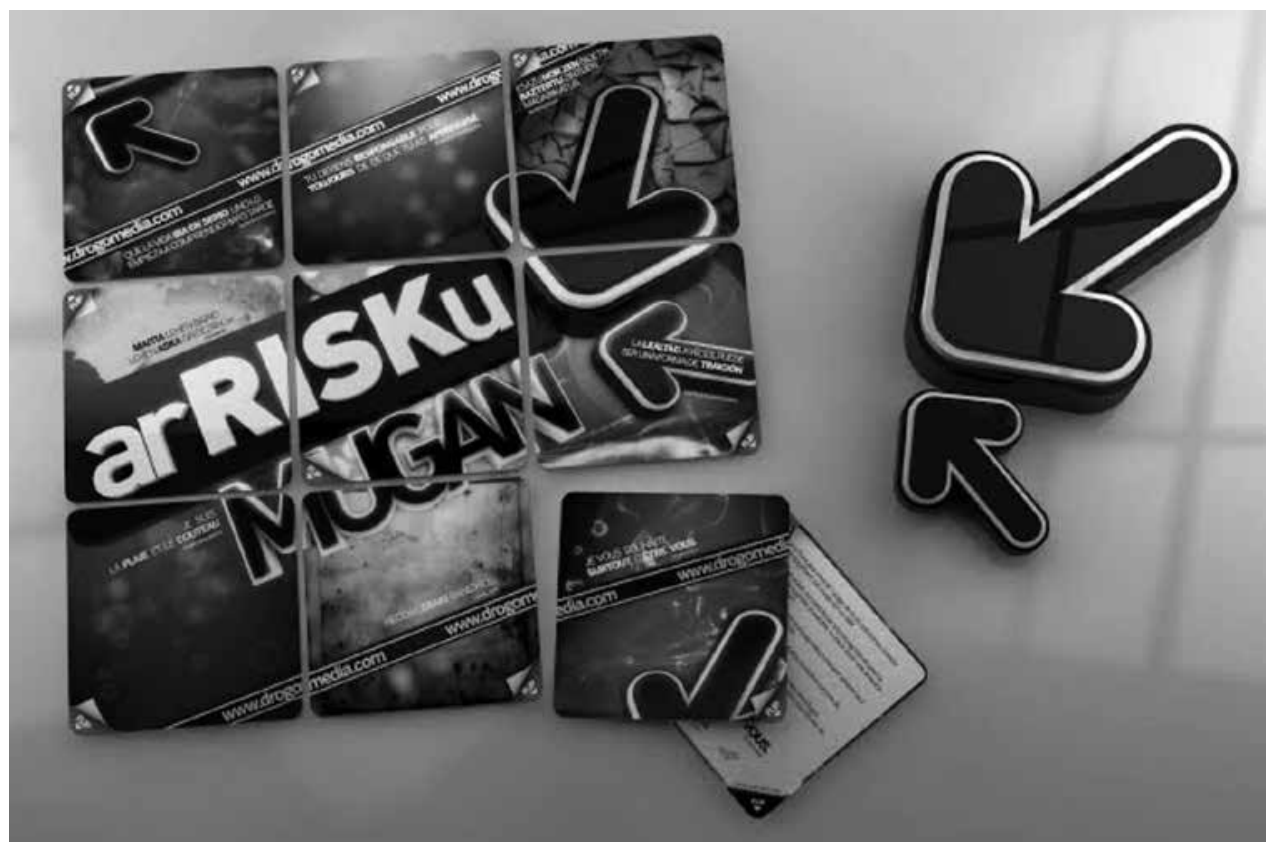

Son muchas las posibilidades que ofrece este proyecto, resultado de un trabajo comunitario enfocado hacia la reducción de riesgos asociados al consumo de drogas, para la zona fronteriza del Bidasoa, por sus particularidades culturales, sociales y políticas. Se apoya en el fuerte interés que ha despertado el tema en todos los agentes sociales de la zona, y está respaldado por la alta motivación hacia su puesta en marcha, y por el contenido y el diseño de los materiales, de alta calidad, consensuado por todos los agentes comunitarios representativos.

Se destaca la importancia de unir los materiales que se distribuyen en las zonas de ocio, con otros de acceso más íntimo y privado, como son las páginas web, en las 
cuales cada persona puede detenerse en contenidos más específicos. Así, se valora la necesidad de que el material vaya asociado a una URL, desde la cual se pudiera acceder a más información. Dicha página web debería estimular a los/as jóvenes para aumentar sus conocimientos sobre las drogas y la reducción de los riesgos asociados a su consumo, basados en la evidencia científica, a través de dinámicas, juegos, concursos, sorteos, etc. Se valoró que la página web asociada debería ser la del Gobierno Vasco y por eso en el diseño final se incluyó la URL correspondiente.

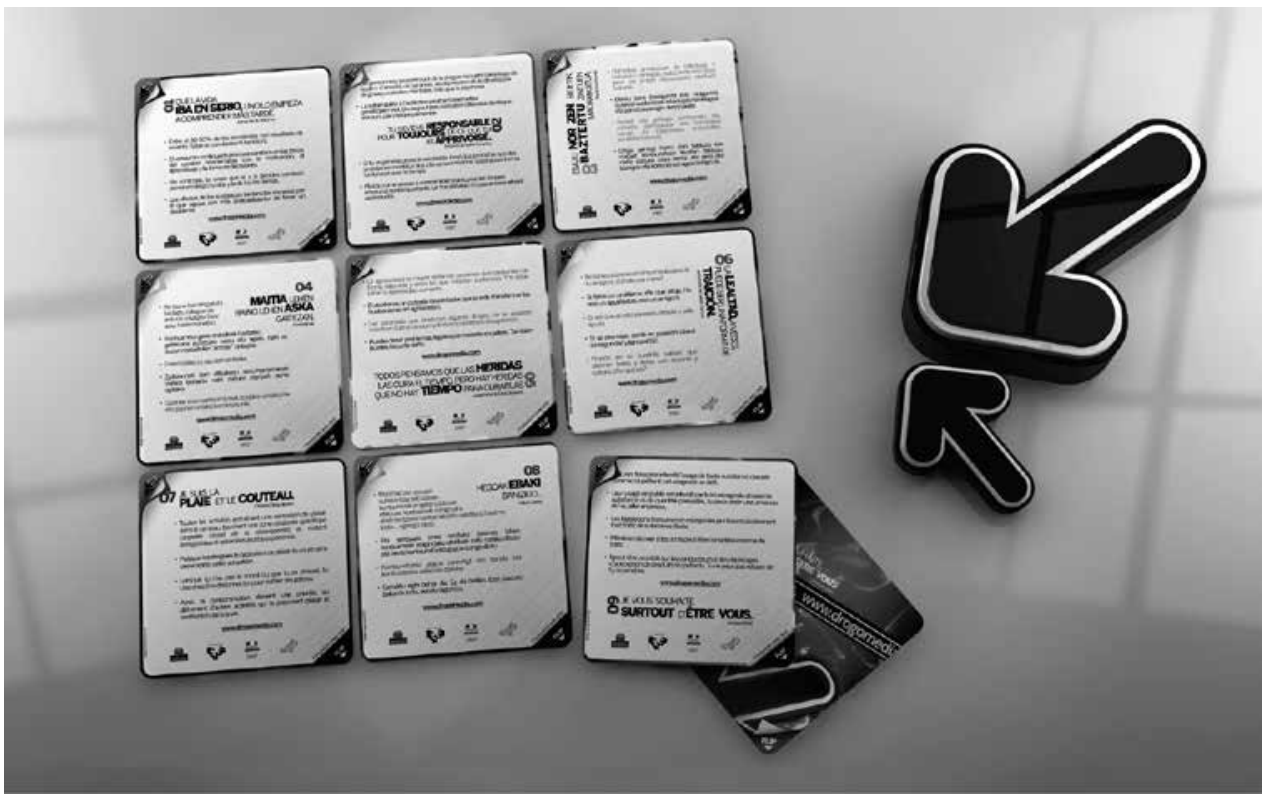

Desde la perspectiva comunitaria, los agentes sociales que han participado en la evaluación del material, deberían participar también de forma activa en el mantenimiento de dicha página web, por lo que se debería crear un mecanismo de valoración permanente de la misma.

Este tipo de Programa se beneficiaría de una distribución progresiva de los materiales, ya que la aparición de los 9 posavasos de forma invasiva en los espacios de ocio y tiempo libre, implicaría perder información importante por saturación. Así se sugiere que la aparición de cada posavasos pudiera ir acompañada de algún acto público reflejado en la prensa local, la radio o la televisión, de forma que el mensaje llegue de forma más amplia a la población. Además, se considera interesante que desde cada municipio se pudieran realizar actividades concretas, con cada uno de los temas que se tratan en los posavasos, llevadas a cabo por técnicos de los Ayuntamientos participantes. 
Los tres objetivos específicos que se plantearon en el proyecto ArRISKu MUGAN se han conseguido, y la evaluación realizada de cada uno de los Planes de acción (40) ha demostrado su adecuación y coherencia con el objetivo general.

Asimismo, las consideraciones transversales para todos los programas de prevención de drogodependencias recomendadas por el Observatorio Europeo de las Drogas y las Toxicomanías (EMCDDA) en sus estándares de calidad (41), han sido tenidas en cuenta; de tal manera que se puede considerar al proyecto arRISKu MUGAN un proyecto sostenible, comunitario, que tiene en cuenta la formación de quienes participan en él, y que ha cuidado en todo su desarrollo cumplir con los principios éticos exigibles en la prevención de drogas.

Las autoras no han podido ser artífices del lanzamiento público del programa y de la distribución de los posavasos en los bares y discotecas del espacio transfronterizo, al depender ambas acciones de Instituciones públicas ajenas a la Universidad.

\section{BIBLIOGRAFÍA}

(1) Insúa P, Lledó M. Consumo de alcohol y otras drogas en los contextos festivos a ambos lados del Bidasoa. Diagnóstico y propuestas de actuación. Donostia-San Sebastián: Universidad del País Vasco/Euskal Herriko Unibertsitatea, 2010.

(2) Laespada T. Las lonjas juveniles serán los txokos del siglo XXI. www.elcorreo.com 2008; [consultado 8-08-2013]: Disponible en: http://www.elcorreo.com/alava/20080630/vizcaya/ lonjas-juveniles-seran-txokos-20080630.html

(3) Webster R, Goodman M, Grainne W. Safer Clubbing. Guidance for licensing authorities, club managers and promoters. Drug Prevention Advisory Service. London: Home Office, 2002.

(4) Insúa P, Lledó M. Plan transfronterizo: Intervención frente al consumo de alcohol y otras drogas a ambos lados del Bidasoa. Norte de Salud Mental 2012; 10 (Suppl 42): 18-29.

(5) Harm Reduction International 2010. What is Harm Reduction? A position statement from the International Harm Reduction Association [consultado 6-8-2012]: Disponible en: http:// www.ihra.net/what-is-harm-reduction.

(6) Salvador T, Del Pozo J. Drogas y Atención Primaria. Guía práctica de intervención sobre el abuso de alcohol y otras drogas. Gobierno de La Rioja. Consejería de Salud. Dirección General de Salud Pública y Consumo. Servicio de Drogodependencias, 2010.

(7) Becoña, E. Bases científicas que sustentan la Prevención de Drogodependencias. Madrid: PNSD, 2002. [consultado 8-8-2013]: Disponible en: http://www.pnsd.msssi.gob.es/Categoria2/ publica/pdf/Bases_cientificas.pdf

(8) Prácticas Óptimas. Tratamiento y rehabilitación de la juventud con problemas de consumo de drogas. Currie JC para Canada's Drug Strategy Division Health Canada, 2001 [consultado 10-8-2012]: Disponible en: http://www.cat-barcelona.com/pdf/biblioteca/adicciones/4_-_best_pract_youth_sp.pdf.

(9) Insúa P y Grijalvo J. Programas de reducción de riesgos en atención a las drogodependencias: Conceptos y acciones específicas. Papeles del Psicólogo, 2000, 77, 33-45 
(10) Juan M, Calafat A, Duch MA, Blay N, Tejera E, Mayol, C. Ocio Nocturno en las Illes Balears. Diagnóstico y propuestas de calidad desde la Salud Pública. Madrid: Plan Nacional sobre Drogas, 2010.

(11) Webster R, Goodman M, Grainne W. Safer Clubbing. Guidance for licensing authorities, club managers and promoters. Drug Prevention Advisory Service. London: Home Office, 2002.

(12) Bellis MA, Hughes K. Consumo recreativo de drogas y reducción de daños en la vida nocturna global. Adicciones 2003; 15 (Suppl 2): 289-305.

(13) Rodríguez Martos A. Guía de estrategias preventivas para reducir la conducción bajo los efectos del alcohol y otras sustancias psicoactivas. Ministerio de Sanidad y Consumo. Delegación del Gobierno para el Plan Nacional sobre Drogas, 2007.

(14) Izquierdo A, Betancourt A. Modelos de intervención comunitaria en salud, República Dominicana. Cuba: Universidad de Ciencias Médicas de Guantánamo [revista electrónica] 2010; (1) [consultado 8-08-2013]: Disponible en: en http://www. monografías.com.

(15) Fernández C, Calafat A, Juan M, Becoña E. Cómo el propio consumo de drogas de los mediadores recreativos tiene implicaciones preventivas. Adicciones 2005; 17 (Suppl 2): 145-55.

(16) European Monitoring Center for Drugs and Drug Addiction (EMCDDA). Risk assessment of new psychoactive substances. Luxembourg: The Publications Office of the European Union, 2009.

(17) Charles M. Local consequences of international drug control. Eguzkilore: Cuaderno del Instituto Vasco de Criminología 2005; 19: 47-54.

(18) Calafat A. et al. Tourism, nightlife and violence: a cross cultural analysis and preventive recommendations. European Commission. Directorate-General Justice. Palma de Mallorca: Irefrea, 2011.

(19) Guía Práctica para una Prevención Eficaz. ADES. 2004 [consultado 10-8-2013]: Disponible en: http://www.pnsd.msssi.gob.es/Categoria2/publica/pdf/prevencion_eficaz.pdf

(20) Prevention and Evaluation Resources Kit (PERK). [consultado 10-8-2013]: European Monitoring Centre for Drugs and Drug Addiction. Disponible en: http://www.emcdda.europa.eu/publications/perk

(21) Cortes I. El desarrollo de los Planes comunitarios en Cataluña. Portularia 2004; 4: 349356.

(22) Marlatt A. Harm Reduction. Pragmatics Strategies for Managing High-Risk Behaviors. London: Guilford Press, 1998

(23) Kumar V, Shah D. Estrategias "push” y “pull” en Internet. Harvard Deusto Marketing y Ventas 2005; 68: 18-25.

(24) Watzlawick P. Teoría de la Comunicación Humana. Barcelona: Herder, 1989.

(25) Márquez Jiménez M A. Las comunicaciones nuestras de cada día. Documentos de trabajo social: Revista de trabajo y acción social 2005; 36: 7-28.

(26) Clark, H.H. y Wilkes-Gibbs, D. (1986). Referring as a colaborative process. Cognition $22,1-39$

(27) Comisión Clínica de la Delegación del Gobierno para el Plan Nacional sobre Drogas. Ministerio de Sanidad y Política Social. Alcohol. Informe $N^{\circ} 2$ de la Comisión Clínica (Madrid) 2007; (1) [consultado 12-08-2013]: Disponible en: http://www.pnsd.msc.es/Categoria2/publica/publicaciones/home.htm 
(28) Comisión Clínica de la Delegación del Gobierno para el Plan Nacional sobre Drogas. Ministerio de Sanidad y Política Social. Cocaína. Informe $N^{\circ} 3$ de la Comisión Clínica (Madrid) 2008; (1) [consultado 12-08-2013]: Disponible en: http://www.pnsd.msc.es/Categoria2/publica/publicaciones/home.html

(29) Comisión Clínica de la Delegación del Gobierno para el Plan Nacional sobre Drogas. Ministerio de Sanidad y Política Social. Cannabis II Informe No 4 de la Comisión (Madrid) 2009; (1) [consultado 9-08-2013]: Disponible en:http://www.pnsd.msc.es/Categoria2/publica/publicaciones/ home.htm

(30) Guardia J, (Ed.). Alcoholismo. Guías Clínicas Basadas en la evidencia científica. Barcelona: Sociodrogalcohol, 2007; (1) [consultado 5-08-2013]: Disponible en: www.sociodrogalcohol. org

(31) Terán A, et al. Cocaína. Guías Clínicas Basadas en la evidencia científica 2008; (1) [consultado 6-08-2013]: Disponible en: www.sociodrogalcohol.org.

(32) Arendt M. Cannabis induced psychosis and subsequent schizophrenia-spectrum disorders: follow-up study of 535 incident cases. British Journal of Psychiatry, 2005; 18, 6, 510-515.

(33) Frisher M, Crome I, Martino O, y Croft P. Assessing the impact of cannabis use on trends in diagnosed schizophrenia in the United Kingdom from 1996 to 2005. Schizophrenia Research, 2009; 113, 2-3, 123-128

(34) Carter A, Capps B, Hall W. Addiction neurobiology. Ethical and social implications. European Monitoring Centre for Drugs and Drug Addiction. Lisboa: Office for Official Publications of the European Communities, 2009.

(35) Rodhes T, Lilly R, Fernández C, Giorgino E, Kemmesis UE, Oseebaard HC et al. Risk factors associated with drug use: the importance of "risk environment". Drugs: Education, prevention and policy, 2003, 10,4,303-329

(36) El Bassel N, Terlikbaeva A y Pinkham S. HIV and Women who use drugs: Double neglect, double risk. The Lancet 2010, 376, 312-314.

(37) Álvarez García F . El delito de tráfico de drogas. Valencia: Tirant lo Blanch, 2009.

(38) Quintero Olivares G, (Ed.). Comentarios a la Parte Especial del Derecho Penal. $7^{\mathrm{a}}$ edición. Navarra: Aranzadi, 2008.

(39) Burkhart G. Selective prevention: First overview on the European situation. Lisboa: EMCDDA, 2003.

(40) Insúa P, (Ed.). Plan transfronterizo: Intervención frente al consumo de alcohol y otras drogas en los contextos festivos a ambos lados del Bidasoa. Informe de investigación no publicado. 2010. Disponible en: https://addi.ehu.es/

(41) EMCDDA: European drug prevention quality standards: a quick guide. 2013 [consultado 10-08-2013]: Disponible en: http://www.emcdda.europa.eu/publications/adhoc/prevention-standard. 